\title{
Association of ADHD with Genetic Variants in the 5'-Region of the Dopamine Transporter Gene: Evidence for Allelic Heterogeneity
}

\author{
K.J. Brookes, ${ }^{1}$ X. Xu, ${ }^{1}$ R. Anney, ${ }^{2}$ B. Franke, ${ }^{3}$ K. Zhou, ${ }^{1}$ Wai Chen, ${ }^{1}$ T. Banaschewski, ${ }^{4}$ J. \\ Buitelaar, ${ }^{3}$ R. Ebstein, ${ }^{5}$ J. Eisenberg, ${ }^{6}$ M. Gill, ${ }^{2}$ A. Miranda, ${ }^{7}$ R.D. Oades, ${ }^{8}$ H. Roeyers, ${ }^{9}$ A. \\ Rothenberger, ${ }^{4}$ J. Sergeant, ${ }^{10}$ E.J.S. Sonuga-Barke, ${ }^{11}$ H.-C. Steinhausen, ${ }^{12}$ E.A. Taylor, ${ }^{1}$ S.V. \\ Faraone, ${ }^{13}$ and P. Asherson ${ }^{1 *}$
}

\section{American Journal of Medical Genetics Part B 147B: 1519-1523}

This is the reformatted manuscript submitted - prior to publication in its final form at DOI 10.1002/ajmg.b.30782

1 MRC Social Genetic Developmental and Psychiatry Centre, Institute of Psychiatry, London, UK

2 Department of Psychiatry, Trinity Centre for Health Sciences, St James's Hospital, Dublin, Ireland

3 Department of Psychiatry, Radboud University, Nijmegen Medical Center, Nijmegen, The Netherlands

4 Child and Adolescent Psychiatry, University of Gottingen, Gottingen, Germany

5 S Herzog Memorial Hospital, Jerusalem, Israel

6 ADHD Clinic, Geha Mental Health Center, Petak-Tikvah, Israel

7 Department of Developmental and Educational Psychology, University of Valencia, Valencia, Spain

8 University Clinic for Child and Adolescent Psychiatry, Essen, Germany

9 Department of Experimental Clinical Health Psychology, Ghent University, Ghent, Belgium

10 Vrije Universiteit, De Boelelaan, Amsterdam, The Netherlands

11 School of Psychology, University of Southampton, Highfield, Southampton, UK

12 Department of Child and Adolescent Psychiatry, University of Zurich, Zurich, Switzerland

13 Departments of Psychiatry and Neuroscience and Physiology, SUNY Upstate Medical University, Syracuse, New York, USA

* Correspondence: E-mail: p.asherson@iop.kcl.ac.uk

\section{Abstract:}

Multiple studies have reported an association between attention deficit hyperactivity disorder (ADHD) and the 10-repeat allele of a variable number tandem repeat (VNTR) polymorphism in the 30-untranslated region (30UTR) of the dopamine transporter gene (DAT1). Yet, recent meta-analyses of available data find little or no evidence for this association; although there is strong evidence for heterogeneity between datasets. This pattern of findings could arise for several reasons including the presence of relatively rare risk alleles on common haplotype backgrounds or the functional interaction of two or more loci within the gene. We previously described the importance of a specific haplotype at the 30 end of DAT1, as well as the identification of associated single nucleotide polymorphisms (SNPs) within or close to 50 regulatory sequences. In this study we replicate the association of SNPs at the 50 end of the gene and identify a specific risk haplotype spanning the 50 and 30 markers. These findings indicate the presence of at least two loci associated with ADHD within the DAT1 gene and suggest that either additive or interaction effects of these two loci on the risk for ADHD. Overall these data provide further evidence that genetic variants of the dopamine transporter gene confer an increased risk for ADHD.

Key Words: attention deficit hyperactivity disorder (ADHD); dopamine transporter gene (DAT1); association study

[Additional supporting information is in the online version of this article.]

Grant sponsor: NIH R01MH62873 


\section{INTRODUCTION:}

Attention deficit hyperactivity disorder (ADHD) is one of the most common and highly heritable behavioral disorders in children. The disorder is characterized by the childhood onset of age inappropriate and impairing levels of hyperactivity, impulsivity and inattention (American Psychiatric Association, 2000) that persists into adult life in around $65 \%$ of cases, either as the full-condition or in partial remission with persistence of symptoms associated with significant clinical impairments [Faraone et al., 2006]. The risk to siblings of ADHD probands is around four- to fivefold the population risk and heritability is estimated to be around 76\% [Faraone et al., 2005]. The search for genetic variants that increase the risk for ADHD has focused mainly on the neurotransmitter systems involved in the response of ADHD symptoms to dopaminergic and noradrenergic medications. Genetic associations have been clearly demonstrated between ADHD and a variable number tandem repeat (VNTR) polymorphism in the dopamine D4 receptor gene and a simple sequence repeat polymorphism that lies upstream to the dopamine D5 receptor gene [Li et al., 2006]. Several other genes involved in the regulation of catecholamine neurotransmission are associated with ADHD in three or more studies and show small yet significant evidence of association [Asherson, 2004; Faraone et al., 2005].

One of the most interesting findings replicated in several studies is the association between the 10-repeat allele of a VNTR located within the 30untranslated (30UTR) region of the dopamine transporter gene (DAT1). The dopamine transporter is the main site of action of stimulant medications, which provide a marked and rapid reduction in the level of ADHD symptoms. Yet a recent study found no overall effect in a comprehensive meta-analysis of available association data [Li et al., 2006]. Despite this, the association remains of considerable interest due to the number of positive reports and the presence of significant evidence of heterogeneity across the datasets, indicating that a subset of datasets may show true association [Li et al., 2006]. One potential cause of heterogeneity could occur if only a subset of individuals carrying the 10repeat allele is at risk for ADHD. This could arise if the DAT1 risk allele interacts with additional genetic or environmental risk factors, which vary in frequency in different populations. For example data from several authors have indicated potential interactions with prenatal environmental risks [Kahn et al., 2003; Brookes et al., 2006a; Neuman et al., 2007].

An alternative explanation, supported by recent data, is that the 10-repeat allele is not the causative allele itself, but rather 'tags' a nearby functional variant that is only partially correlated with the 10repeat allele, or that the 10 -repeat allele interacts with a second locus within the DAT1 gene [Asherson et al., 2007]. Evidence for this comes from the observation in several studies that specific DAT1 haplotypes containing the 10-repeat allele confer risk for ADHD [Barr et al., 2001; Greenwood et al., 2002; Hawi et al., 2003; Galili-Weisstub et al., 2005; Brookes et al., 2006a]. A key finding from our own research was the observation in two independent samples from the UK and Taiwan of association between ADHD and a specific haplotype of the 10-repeat allele with the 6-repeat allele of a VNTR located in intron 8 [Brookes et al., 2006a]. This finding was subsequently replicated in the first set of samples (ST1) from the International Multi-centre ADHD Genetics (IMAGE) project, using a large sample of ADHD combined subtype cases [Asherson et al., 2007]. 
In an earlier study, the IMAGE ST1 samples were also screened for association with 32 SNPs and two VNTRs spanning DAT1, as part of a study that included the analysis of 51 candidate genes [Brookes et al., 2006b]. None of the markers were significant when adjusted for the number of markers investigated, but we reported significant gene-wide association for the sum of chi-squares for SNP associations across the gene. The analysis of two additional VNTR markers located within introns 3 and 14 was subsequently completed. To summaries the data from ST1, we found 6 SNP markers and 3 VNTR markers showing nominal evidence of association with combined type ADHD ( $P \leq 0.05$, Table 1$)$. The association signal fell into two groups of markers at the 30 and 50 ends of the gene. Genetic variants within each region were found to be in high linkage disequilibrium (LD), but there was low LD between the two regions, suggesting the presence of two independent loci associated with ADHD. In this study we extend these findings by investigating the association of four SNP markers in the 30 and 50 region (two from each region) that were associated with ADHD in the ST1 sample, in a second set of samples (ST2) collected by the IMAGE consortium. We further analyze the entire dataset and look for evidence of additive effects between the two ends of the gene.

\section{Methods:}

\section{The IMAGE Sample}

European Caucasian subjects were recruited from 12 specialist clinics in 8 countries: Belgium, Germany, Holland, Ireland, Israel, Spain, Switzerland, and United Kingdom. Ethical approval for the study was obtained from National Institute of Health registered ethical review boards for each centre. Detailed information sheets were provided and informed consent obtained from the majority of children and from all of their parents. All ADHD probands and their siblings were aged 5-17 at the time of entry into the study and access was required to one or both biological parents for DNA collection. Entry criteria for probands were a clinical diagnosis of DSMIV combined subtype ADHD and having one or more full siblings available for ascertainment of clinical information and DNA collection. Exclusion criteria applying to both probands and siblings include autism, epilepsy, $1 Q<70$, brain disorders and any genetic or medical disorder associated with externalizing behaviors that might mimic ADHD. Inclusion criteria included white European ancestry and living at home with at least one biological parent.

The diagnosis of ADHD was made following a parent interview with the Parental Account of Child Symptoms interview [PACS; Taylor et al., 1986] that asks about ADHD symptoms in various settings. An algorithm was used to derive each of the DSM-IV ADHD symptoms from the PACS interview data and these were combined with items that scored two or more from teacher ratings of DSM-IV items taken from the long version of the Conners' Teacher Rating Scale [Conners, 1995]. The diagnosis of ADHD was made if sufficient items were identified to fulfill DSM-IV criteria, and both impairment (based on severity of symptoms identified in the PACS interview) and pervasiveness (based on the presence of ADHD symptoms in more than one setting from PACS and scoring more than one item on the teacher Connors) were present. In 28 cases where no Connors data was present pervasiveness was defined on the basis of PACS data alone [Brookes et al., 2006b]. Further details on the clinical evaluation of the ADHD probands can be found in the original study of 51 candidate genes in the ST1 sample [Brookes et al., 2006b]. 
Table 1: Markers within the IMAGE stage 1 dataset (ST1) displaying nominal significance $(\mathrm{P} \leq 0.056)$ for association with ADHD

\begin{tabular}{l|llllllll}
\hline Marker & Position (bp) & Location & MAF & P value & Allele & T & NT & OR \\
\hline 3'UTR VNTR & - & 3'UTR & 0.29 & 0.03 & 10-repeat & 277 & 245 & 1.13 \\
\hline Intron 14 VNTR & - & Intron 14 & 0.07 & 0.06 & Insertion & 106 & 80 & 1.33 \\
\hline rs40184 & 144,8077 & Intron 13 & 0.44 & 0.005 & G & 349 & 278 & 1.26 \\
rs3776513 & 146,0104 & Intron 10 & 0.18 & 0.05 & C & 183 & 147 & 1.24 \\
Intron 8 VNTR & - & Intron 8 & 0.22 & 0.006 & 6-repeat & 243 & 207 & 1.17 \\
rs2652511 & 149,9389 & 5' Flanking & 0.41 & 0.02 & G & 353 & 293 & 1.20 \\
rs11564750 & 150,0762 & 5' Flanking & 0.09 & 0.03 & $\mathrm{G}$ & 127 & 94 & 1.35 \\
rs10070282 & 150,3444 & 5' Flanking & 0.42 & 0.01 & $\mathrm{~A}$ & 358 & 294 & 1.22 \\
rs2550946 & 150,3513 & 5' Flanking & 0.42 & 0.007 & $\mathrm{G}$ & 358 & 289 & 1.24 \\
\hline
\end{tabular}

\section{Stage 2 (ST2) Sample}

\section{and Combined ST1pST2 Sample}

We previously reported DAT1 genotype data from the analysis of the ST1 sample, which consisted of 776 DSM-IV combined type ADHD cases with DNA from both parents available for $90 \%$ of families and from one parent in $7 \%$ of families [Brookes et al., 2006b]. In this study we report on new data from the ST2 sample consisting of a further 435 combined type probands (376 probands plus 59 affected siblings). DNA was available from both parents in $80 \%$ of the families and from one parent in $19.2 \%$ of the families. Following the accumulation of further clinical information and a comprehensive audit of the entire clinical and DNA data sets completed in April 2007, the final set of individuals with combined type ADHD and fulfilling all inclusion and exclusion criteria in ST1 and ST2 were amended: the final combined ST1 and ST2 dataset used in this study consisted of 1,147 individuals with DSM-IV combined subtype ADHD from 988 affected probands and 150 of their affected siblings.

\section{Genotyping and SNP Selection}

The initial scan of ST1 samples identified two clusters of associated SNP markers at the 30 and 50 ends of the gene [Brookes et al., 2006b]. From these we selected two SNPs from the 3' region (rs40184 and rs3776513) and two located in the 50 region (rs2550946 and rs11564750). Since three of the associated 50 SNPs in the ST1 study were found to be in strong LD with an average r2 of 0.97 (rs2622511, rs2550946, rs10070282) we selected only one (rs2550946) for analysis in ST2, whereas the other marker we selected ( $r$ 11564750) was only weakly associated with this group of SNPs (average $r^{2}$ of 0.14 ). Genotyping of the 3'UTR and intron 8 VNTRs were previously carried out in the ST2 sample and the results reported elsewhere [Asherson et al., 2007].

The SNP markers were genotyped using the ABI SNPlex [Tobler et al., 2005] and $A B I$ TaqMan genotyping protocols (Applied Biosystems, Foster City, CA). The selected markers were initially analyzed on the ABI SNPlex system. Due to a high failure rate with the SNPlex assays, genotypes were repeated using the $A B I$ TaqMan assay. We found no discrepancies in overlapping genotypes using the two methods. Standard protocols provided by $A B I$ were followed for both types of assays. Three SNP assays were designed for the TaqMan 7900HT: SNP rs40184 was available from 'assayson-demand', whilst SNP markers rs11564750 and rs2550946 were custom designed for the project. We were unable to design a TaqMan assay for SNP rs3776513. 


\section{Analysis}

The transmission disequilibrium test was performed for probands with the combined subtype of ADHD using the UNPHASED program (TDTPHASE; [Dudbridge, 2003, www.rfcgr.mrc.ac.uk/ fdudbrid/software/unphased]. Nominal evidence for association was determined by P-values less than 0.05 and adjusted significance estimated by permutation tests. In addition we utilized the WHAP program to conduct conditional analyses that test the contribution of genetic variants to haplotype associations (www. genome.wi.mit.edu/shaun/whap). In the conditional test a SNP is dropped from the null model and compared to the alternative model. A significant P-value indicates that the dropped SNP has an independent effect and makes a significant contribution to the haplotype association.

\section{RESULTS:}

\section{LD Across the Gene}

Using the data from the ST1 study, we examined the LD structure across the gene, with values provided from the UNPHASED software package [Genro et al., 2007] (Fig. S1). Observation of $D^{\prime}$ values indicate that there are 4 main LD blocks: one of the LD regions spans the 3'UTR, another spans intron 11 to intron 8, a third spans exon 5 to intron 2 (rs403636) and a fourth spans the 5'UTR and upstream flanking region. These data indicate that the two regions of ADHD associated markers identified at the $3^{\prime}$ and $5^{\prime}$ ends of the gene in the previous study represent independent SNP clusters and cannot be explained by one set of SNPS tagging the other.

\section{Replication Study}

Genotype data from the 4 SNPs in the ST2 dataset showed similar minor allele frequencies to those observed in the ST1 study (Table 2). The 4 SNPs were in Hardy-
Weinberg equilibrium and had a genotype success rate of over 97\%; except for rs3776513, which could not be genotyped on the TaqMan platform and only $77 \%$ of the ST2 genotypes could be reliably called from the SNPlex assay. TDT analysis found that 2 of the 4 SNP markers showed nominal significance $(p<0.01)$ and surpassed the Bonferroni correction for the 4 markers tested: rs11564750 located in the $5^{\prime}$ promoter region and rs3776513 located within intron 1' (Table 2). For both these SNP markers the same allele was preferentially transmitted in both the ST1 and ST2 datasets. The remaining 2 SNP markers rs2550946 (50-region), and rs40184 (intron 13) were not significantly associated, however both SNP markers showed over-transmission of the same allele observed to be the risk allele in the ST1 dataset. In the combined analysis of the ST1 and ST2 datasets, nominally significant associations were observed for all 4 SNP markers with estimated odds ratios in the range 1.2-1.5.

\section{Haplotype Analysis and Conditional Tests}

Haplotype analysis was performed for the 4 markers genotyped in both the ST1 and ST2 datasets (Table 3). Pair-wise haplotype analysis indicated that the most significant finding occurred between rs2550946 in the $5^{\prime}$ promoter region and rs40184 in intron $13(P<0.00002)$. These two markers lie over $55 \mathrm{~kb}$ apart and have an estimated $r^{2}$ of 0.02 for transmitted alleles and an $r^{2}$ of 0.0002 for nontransmitted alleles. The 4-marker haplotype has a global P-value of 0.0001 with only one haplotype (GGGC) displaying significant over transmission to ADHD probands (haplotype specific $p$-value $=$ $\left.1.8 \times 10^{-6}\right)$ : these alleles coincide with those transmitted in the single marker analysis (Table 4).

WHAP analysis confirmed the haplotype association, but with reduced significance using the permutation routine 
Table 2.

Findings from TDT Analysis of the ST1, and ST2 Datasets Independently and When Combined

\begin{tabular}{|c|c|c|c|c|c|c|c|c|c|c|}
\hline SNP ID & $\begin{array}{l}\text { BP } \\
\text { Position }\end{array}$ & Location & Dataset & $\begin{array}{l}\text { Minor } \\
\text { Allele }\end{array}$ & & $\begin{array}{l}\text { TDT } \\
\text { P value }\end{array}$ & $\begin{array}{l}\text { Risk } \\
\text { Allele }\end{array}$ & $T$ & NT & OR \\
\hline rs2550946 & 1503513 & $\begin{array}{l}\text { ' } \\
\text { Promoter }\end{array}$ & $\begin{array}{l}\text { ST1 } \\
\text { ST2 } \\
\text { ST1 \& } \\
\text { ST2 }\end{array}$ & $\begin{array}{l}\mathrm{A} \\
\mathrm{A} \\
\mathrm{A}\end{array}$ & $\begin{array}{l}0.42 \\
0.41 \\
0.42\end{array}$ & $\begin{array}{l}\mathbf{0 . 0 0 6} \\
0.45 \\
0.01\end{array}$ & $\begin{array}{c}\mathrm{G} \\
\mathrm{G} \\
\mathrm{G}\end{array}$ & $\begin{array}{l}358 \\
131 \\
485\end{array}$ & $\begin{array}{l}289 \\
119 \\
408\end{array}$ & $\begin{array}{l}1.24 \\
1.1 \\
1.19\end{array}$ \\
\hline rs11564750 & 1500762 & $\begin{array}{l}5 \\
\text { Promoter }\end{array}$ & $\begin{array}{l}\text { ST1 } \\
\text { ST2 } \\
\text { ST1 \& } \\
\text { ST2 }\end{array}$ & $\begin{array}{l}\mathrm{C} \\
\mathrm{C} \\
\mathrm{C}\end{array}$ & $\begin{array}{l}0.09 \\
0.1 \\
0.09\end{array}$ & $\begin{array}{l}0.03 \\
0.004 \\
0.0006\end{array}$ & $\begin{array}{c}\mathrm{G} \\
\mathrm{G} \\
\mathrm{G}\end{array}$ & $\begin{array}{l}127 \\
67 \\
195\end{array}$ & $\begin{array}{l}94 \\
38 \\
133\end{array}$ & $\begin{array}{l}1.35 \\
1.76 \\
1.47\end{array}$ \\
\hline rs3776513 & 1460104 & Intron 10 & $\begin{array}{l}\text { ST1 } \\
\text { ST2 } \\
\text { ST1 \& } \\
\text { ST2 }\end{array}$ & $\begin{array}{l}\mathrm{T} \\
\mathrm{T} \\
\mathrm{T}\end{array}$ & $\begin{array}{l}0.18 \\
0.21 \\
0.19\end{array}$ & $\begin{array}{l}0.05 \\
0.01 \\
0.009\end{array}$ & $\begin{array}{c}G \\
G \\
G\end{array}$ & $\begin{array}{l}183 \\
80 \\
247\end{array}$ & $\begin{array}{l}147 \\
51 \\
193\end{array}$ & $\begin{array}{l}1.24 \\
1.57 \\
1.28\end{array}$ \\
\hline rs40184 & 1448077 & Intron 13 & $\begin{array}{l}\text { ST1 } \\
\text { ST2 } \\
\text { ST1 \& } \\
\text { ST2 }\end{array}$ & $\begin{array}{l}\mathrm{T} \\
\mathrm{T} \\
\mathrm{T}\end{array}$ & $\begin{array}{l}0.44 \\
0.46 \\
0.45\end{array}$ & $\begin{array}{l}\mathbf{0 . 0 0 5} \\
0.31 \\
0.005\end{array}$ & $\begin{array}{l}\mathrm{C} \\
\mathrm{C} \\
\mathrm{C}\end{array}$ & $\begin{array}{l}349 \\
149 \\
498\end{array}$ & $\begin{array}{l}278 \\
132 \\
414\end{array}$ & $\begin{array}{l}1.26 \\
1.13 \\
1.2\end{array}$ \\
\hline
\end{tabular}

$\mathrm{MAF}=$ minor allele frequency.

Table 3:

Significance values for 2-marker haplotypes for the SNP markers investigated in the combined ST1 and ST2 dataset are located in the upper triangle. Linkage Disequilibrium values determined by $\mathrm{r}^{2}$ are present in the lower triangle.

\begin{tabular}{|l|llll|}
\hline & rs2550946 & rs11564750 & rs3776513 & rs40184 \\
\hline rs2550946 & & $\mathbf{0 . 0 0 0 4}$ & $\mathbf{0 . 0 0 4}$ & $\mathbf{0 . 0 0 0 0 2}$ \\
rs11564750 & 0.16 & & $\mathbf{0 . 0 0 0 4}$ & $\mathbf{0 . 0 0 0 2}$ \\
rs3776513 & 0.0004 & 0.05 & & $\mathbf{0 . 0 1}$ \\
rs40184 & 0.02 & 0.002 & 0.08 & \\
\hline
\end{tabular}

( $p=0.004)$. When testing the effect of dropping each marker from the two marker haplotypes, a significant difference of fit was observed for both $\mathrm{rs} 40184(\mathrm{p}=$ 0.008 ) and $r s 2550946$ ( $p=0.03$ ). These findings indicate that independent markers at the $3^{\prime}$ and $5^{\prime}$ ends of the genes both contribute significantly to the haplotype association. In contrast, when the two 3' SNP marker and the two 5' SNP marker haplotypes were examined it was found that one of the two markers from each group could be dropped from the analysis, indicating that no additional information was obtained from the analysis of haplotypes within the $3^{\prime}$ and within the 5' SNP clusters.

\section{DISCUSSION}

There are two main novel findings in this study. First, we replicate earlier evidence of association with between ADHD and SNP markers that span genetic variation in the $5^{\prime}$ regulatory region of DAT1, including the promoter region. Second we show that markers that form independent SNP clusters at the 3' and 5' ends of the gene contribute significantly 
Table 4:

The transmission of alleles in a four marker haplotype, only one risk haplotype is observed (in red)

\begin{tabular}{|l|l|l|l|r|r|r|}
\hline rs2550946 & rs11564750 rs3776513 & rs40184 & T & IIT & OR \\
\hline \hline A & C & G & C & 43 & 54 & 0.80 \\
\hline A & C & G & T & 26 & 29 & 0.90 \\
\hline A & C & T & C & 0 & 9 & 0.00 \\
\hline A & C & T & T & 5 & 12 & 0.42 \\
\hline A & G & G & C & 142 & 137 & 1.04 \\
\hline A & G & G & T & 112 & 119 & 0.94 \\
\hline A & G & T & C & 2 & 4 & 0.50 \\
\hline A & G & T & T & 56 & 54 & 1.04 \\
\hline G & C & G & C & 1 & 3 & 0.33 \\
\hline G & C & G & T & 0 & 1 & 0.00 \\
\hline G & C & T & C & 0 & 1 & 0.00 \\
\hline G & G & G & C & 279 & 177 & 1.58 \\
\hline G & G & G & T & 122 & 149 & 0.82 \\
\hline G & G & T & C & 28 & 32 & 0.88 \\
\hline G & G & T & T & 48 & 83 & 0.58 \\
\hline
\end{tabular}

to a specific haplotype association. This is a key finding that suggests two independent functional sites within the DAT1 gene associated with ADHD.

The ST2 IMAGE samples used in this study provide a small but clinically homogenous sample that was ascertained using identical clinical procedures to the ST1 sample. For this reason replication is feasible despite the relatively small sample size of ST2 compared to ST1. The selection of the four SNP markers was based on nominally significant associations observed in the ST1 study [Brookes et al., 2006b] with two SNP markers selected from each of two associated SNP clusters. Significant association with ADHD was observed for two of the markers rs11564750 and rs3776513 located in the 5' promoter region and intron 10 , respectively, which is a direct replication of findings from ST1. Although the other two SNPs were not significantly associated with ADHD in ST2, they showed the same direction of effect for over-transmission of alleles to affected offspring. Analysis of the two SNPs that were not associated with ADHD in ST2 might also lack power, because they had relatively high minor allele frequencies compared to the significantly associated SNPs.

Several other groups have also identified SNP associations with ADHD in the 5' region of DAT1. Genro et al. [2007] using a sample of ADHD probands from Brazil detected association to the same allele of rs2652511 reported in this study. Furthermore, they found that the effect size for this association was greater when they restricted their analysis to the subset of individuals with combined type ADHD. Ohadi et al. [2006] found association of ADHD with the T-allele of a core promoter SNP at position -67 ( $r$ 2975226) which is thought to possess functional properties. The T-allele of this SNP creates a putative SIF-binding element [Rubie et al., 2001] capable of increasing transcription twofold [Greenwood et al., 2002; Greenwood and Kelsoe, 2003]. This putative functional SNP lies $4.8 \mathrm{~kb}$ downstream from (and is in high LD with) SNP rs2550946 that is associated with ADHD in this study; and only 774 bp from SNP rs2652511 that was associated with ADHD in the ST1 study 
[Brookes et al., 2006b]. This SNP also lies in a region of highly conserved sequence identity (63\%) between the mouse and human 5' flanking regions [Donovan et al., 1995]. Finally, Friedel et al. [2007] recently completed a comprehensive analysis of $3^{\prime}$ SNPs spanning the $3^{\prime}$ and $5^{\prime}$ regions of DAT1 in a sample of ADHD cases from Germany. One of the associated markers identified in their study (rs403636) showed the same allelic association to that reported in our ST1 study but did not clearly identify an overlapping haplotype with that reported in this study.

The finding of replicated association with SNPs within the $5^{\prime}$ regulatory region of DAT1 is clearly of key interest and further work is needed to delineate the role of functional variation in this region. Drgon et al. [2006] reported that the rs2550946 is involved in altered transcriptional regulation, with the C-allele promoting Rps3a-2-homol-D transcription factor binding, whereas the T-allele promotes GHFI/Pit1-pr-1 binding. Based on the association with the $\mathrm{C}$-allele in this study the association with ADHD might be mediated by increased binding of the transcription factor Rps3a-2-homol-D in ADHD cases. Drgon et al. [2006] also reported that the $\mathrm{C}$-allele of SNP rs2652511, that was associated with ADHD in this study and is in strong LD with rs2550946, is associated with significantly higher levels of DAT1 expression in vivo.

The second key finding from this study was the contribution of genetic variants to the risk for ADHD from both the 3' and 5' regions. However, it is not clear from the analysis of association data alone what are the genetic sequences within these regions that are the primary source of the association signals. No single marker was responsible for the haplotype association and the evidence from WHAP indicated that the presence of genetic variation in both regions was important. The finding that only a single haplotype spanning the 4 SNP markers in this study was associated with increased risk for ADHD (odds ratio = 1.58, global $p=0.00016$, haplotype specific $p=1.7 \times 10^{-6}$ ) suggests a possible interaction between the two loci, but could also arise from a simple additive effect of two main associated loci. The possibility that risk variants at more than one site are required to enhance the risk for ADHD could contribute to the observed heterogeneity see within metaanalytic studies of DAT1 [Li et al., 2006].

The functional role of the two VNTR markers located within the 3'UTR and intron 8 in our earlier study also remains uncertain. In our previous report we found evidence for a contribution of both loci to a haplotype association between the two VNTRs [Asherson et al., 2007], however it was not possible to distinguish between two possible explanations. First that the two VNTR markers tag a single (unidentified) functional site; and second that there is a functional interaction between two VNTR markers (or closely associated functional variants). It is also feasible that the two VNTRs mark two independent loci with purely additive effects at the level of gene function. We have also analyzed the VNTR markers in relation to the SNPs investigated in this study and found that they formed part of the same 3 ' haplotype reported here (data not shown). We therefore conclude that analysis of DAT1 in the IMAGE data-set suggests the presence of two and potentially three functional loci.

In summary we have replicated association findings between ADHD and genetic variants within DAT1, that were initially identified as nominal associations in a scan of SNP markers tagging genetic variation across 51 candidate genes. Limitations of this study include possible heterogeneity arising from the use of a multi-site European sample, although this 
is largely controlled by the use of the Transmission Disequilibrium Test as our primary tool for association analysis. A further problem was the relatively small sample size of this study compared to the initial ST1 study, which might account for replication of only two out of the four markers studied. The overall findings in the combined IMAGE dataset suggest that there may additive or interactional effects of two or more functional loci within DAT1. Further analysis of the functional consequences of genetic variation within the gene is therefore required and replication and meta-analytic studies in independent datasets are required to confirm or refute the pattern of findings reported here.

\section{ACKNOWLEDGEMENTS}

The IMAGE project is a multi-site, international effort supported by $\mathrm{NIH}$ grant R01MH62873 to S.V. Faraone. Site Principal Investigators are Philip Asherson, Tobias Banaschewski, Jan Buitelaar, Richard P. Ebstein, Stephen V. Faraone, Michael Gill, Ana Miranda, Fernando Mulas, Robert D. Oades, Herbert Roeyers, Aribert Rothenberger, Joseph Sergeant, Edmund Sonuga-Barke, and HansChristoph Steinhausen. Senior coinvestigators are Margaret Thompson, Pak Sham, Peter McGuffin, Robert Plomin, Ian Craig and Eric Taylor. Chief Investigators at each site are Rafaela Marco, Nanda Rommelse, Wai Chen, Henrik Uebel, Hanna Christiansen, U. Mueller, Cathelijne Buschgens, Barbara Franke, Lamprini Psychogiou. We thank all the families who kindly participated in this research.

\section{REFERENCES}

American Psychiatric Association. 2000. Diagnostic and statistical manual of mental disorders version IV text revision. Washington DC: American Psychiatric Association.
Asherson P. 2004. Attention-deficit hyperactivity, disorder in the postgenomic era. Eur Child Adolesc Psychiatry 13(Suppl 1):150-170.

Asherson $P$, et al. 2007. Confirmation that a specific haplotype of the dopamine transporter gene is associated with combined-type ADHD. Am J Psychiatry 164(4):674-677.

Barr CL, et al. 2001. Haplotype study of three polymorphisms at the dopamine transporter locus confirm linkage to attention-deficit/hyperactivity disorder. Biol Psychiatry 49(4):333-339.

Brookes KJ, et al. 2006a. A common haplotype of the dopamine transporter gene associated with attentiondeficit/hyperactivity disorder and interacting with maternal use of alcohol during pregnancy. Arch Gen Psychiatry 63(1):74-81.

Brookes K, et al. 2006b. The analysis of 51 genes in DSM-IV combined type attention deficit hyperactivity disorder: Association signals in DRD4, DAT1 and 16 other genes. Mol Psychiatry 147:9499.

Conners CK. 1995. The Conners Rating Scale: Instruments for the Assessments of Childhood Psychopathology in Duke University.

Donovan DM, et al. 1995. Human and mouse dopamine transporter genes: Conservation of 5'-flanking sequence elements and gene structures. Brain Res Mol Brain Res 30(2):327-335.

Drgon T, et al. 2006. Common human 50 dopamine transporter (SLC6A3) haplotypes yield varying expression levels in vivo. Cell Mol Neurobiol 26(46):875-889.

Dudbridge F. 2003. Pedigree disequilibrium tests for multilocus haplotypes. Genet Epidemiol 25(2): 115-121. 
Faraone SV, et al. 2005. Molecular genetics of attention-deficit/ hyperactivity disorder. Biol Psychiatry 57(11):1313-1323.

Faraone SV, Biederman J, Mick E. 2006. The age-dependent decline of attention deficit hyperactivity disorder: A metaanalysis of follow-up studies. Psychol Med 36(2):159-165.

Friedel S, et al. 2007. Association and linkage of allelic variants of the dopamine transporter gene in ADHD. Mol Psychiatry 12:923-933.

Galili-Weisstub E, et al. 2005. Dopamine transporter haplotype and attentiondeficit hyperactivity disorder. Mol Psychiatry 10(7):617-618.

Genro JP, et al. 2007. A promoter polymorphism $(-839 \quad \mathrm{C}>\mathrm{T})$ at the dopamine transporter gene is associated with attention deficit/ hyperactivity disorder in Brazilian children. Am J Med Genet Part B 144B (2):215-219.

Greenwood TA, Kelsoe JR. 2003. Promoter and intronic variants affect the transcriptional regulation of the human dopamine transporter gene. Genomics 82(5):511-520.

Greenwood TA, et al. 2002. Segmental linkage disequilibrium within the dopamine transporter gene. Mol Psychiatry 7(2):165-173.

Hawi Z, et al. 2003. Linkage disequilibrium mapping at DAT1, DRD5 and DBH narrows the search for $A D H D$ susceptibility alleles at these loci. Mol Psychiatry 8(3):299-308.

Kahn RS, et al. 2003. Role of dopamine transporter genotype and maternal prenatal smoking in childhood hyperactive-impulsive, inattentive, and oppositional behaviors. J Pediatr 143(1):104-110.
Li D, et al. 2006. Meta-analysis shows significant association between dopamine system genes and attention deficit hyperactivity disorder (ADHD). Hum Mol Genet 15:2276-2284.

Neuman RJ, et al. 2007. Prenatal smoking exposure and dopaminergic genotypes interact to cause a severe ADHD subtype. Biol Psychiatry 61(12):13201328.

Ohadi M, et al. 2006. Attentiondeficit/hyperactivity disorder (ADHD) association with the DAT1 core promoter -67T allele. Brain Res 1101:14.

Rubie $\mathrm{C}$, et al. 2001. The human dopamine transporter gene: The $5^{\prime}$-flanking region reveals five diallelic polymorphic sites in a Caucasian population sample. Neurosci Lett 297(2):125-128.

Taylor E, et al. 1986. Conduct disorder and hyperactivity. I. Separation of hyperactivity and antisocial conduct in British child psychiatric patients. $\mathrm{Br} \mathrm{J}$ Psychiatry 149:760-767.

Tobler AR, et al. 2005. The SNPlex genotyping system: $A$ flexible and scalable platform for SNP genotyping. J Biomol Tech 16(4):398-406. 